\title{
Especialização inteligente e redes de conhecimento. A Universidade de Coimbra num contexto de globalização
}

\author{
R. Gama (a), C. Barros (b), R. David (c), R. Fernandes (d) \\ (a) Universidade de Coimbra/CEGOT, rgama@fl.uc.pt \\ (b) Universidade de Coimbra, cbarros@fl.uc.pt \\ (c) Universidade de Coimbra/Faculdade de Letras (GAPCI), gapci2@fl.uc.pt \\ (d) Universidade de Coimbra/CEGOT, r.fernandes@fl.uc.pt
}

\begin{abstract}
Resumo
A especialização inteligente assume-se como uma abordagem central no âmbito da estratégia europeia 2020, procurando fomentar o desenvolvimento intensivo do conhecimento. Utilizando dados dos projetos de investigação do Horizonte 2020, pretende-se avaliar a configuração das redes estabelecidas, tendo em atenção as instituições/organizações envolvidas e a intensidade e expressão territorial das relações identificadas. Privilegia-se a análise de redes sociais e a leitura dos resultados numa dupla dimensão, topológica e territorial. Em paralelo, e para perceber as lógicas da estratégia de investigação da Universidade de Coimbra caracteriza-se o potencial das unidades de I\&D (recursos humanos, participação em projetos, resultados da investigação). A leitura territorial visa identificar as novas dinâmicas associadas à missão da universidade na qualificação regional no quadro da especialização inteligente e da geografia das redes do conhecimento.
\end{abstract}

Palavras chave: Especialização Inteligente, Redes de Conhecimento, Universidade de Coimbra, Desenvolvimento Regional

\section{INTRODUÇÃO}

A evolução que tem vindo a ser observada nos anos mais recentes, tem conduzido a que as estratégias de desenvolvimento económico e territorial, delineadas às escalas europeia e nacional, consagrem especial importância às atividades de investigação e à ciência, assim como à inovação e ao papel dos territórios inovadores para o desenvolvimento (Patrício, 2010; Heitor \& Horta, 2011; Brandão, 2016).

A realização dos objetivos da Estratégia Europa 2020 de crescimento inteligente, sustentável e inclusivo está dependente da investigação e inovação enquanto motores essenciais de prosperidade social e económica e de sustentabilidade ambiental (CE, 2011). A perspetiva do crescimento inteligente valoriza a educação, a investigação e a inovação como elementos que podem garantir, a prazo, o maior retorno do investimento, em termos de ganhos de produtividade, assegurando, também, a necessária capacidade de adaptação dos recursos humanos aos novos desafios económicos (CE, 2010).

A estratégia de especialização inteligente decorre da natureza sistémica frágil para a difusão de conhecimento e tecnologia ao sistema produtivo na União Europeia. Do pondo de vista teórico procede da economia do conhecimento e da mudança tecnológica e visa construir um quadro lógico que permita definir prioridades em termos de política de desenvolvimento dos territórios. Assenta na trajetória dos territórios, num processo de pesquisa empreendedora, num domínio (espaço tecnológico) com uma dimensão relevante e estabelecendo relações (McCann, 2015). A valorização deste conceito no contexto regional pressupõe que se considere a proximidade tecnológica existente no domínio (espaço tecnológico), o grau de imbricação existente entre as diferentes atividades, competências e instituições e, igualmente, as ligações desenvolvidas e aprofundadas entre os diferentes atores (indivíduos, empresas, instituições e outros) na incorporação de conhecimento de diferentes fontes. Trata-se de identificar e perceber de que forma os diferentes tipos de proximidade (geográfica, institucional, organizacional, cognitiva e cultural), associados ao nível de participação de atores de diferentes esferas e competências e à capacidade resultante dos relacionamentos entre atores se organizam visando a procura de novas soluções que incorporem conhecimento e tecnologia nos territórios.

A produção de conhecimento deve ser analisada considerando as condições do contexto em que é gerado e 
a tradução em inovação (nomeadamente económica), sendo que conhecimento e inovação fazem parte de um todo indissociável. As diferentes abordagens têm vindo a considerar os diferentes tipos e objetivos das atividades de ciência, assim como a forma como o conhecimento é produzido. Desta forma, é necessário centrar a discussão nos processos de produção conhecimento, sendo este o recurso essencial para a inovação (Lundvall \& Johnson, 1994). A complexidade destes processos traduz-se em origens cada vez mais dispersas, numa diversidade de atores envolvidos, ao mesmo tempo que os processos são mais distribuídos quanto ao locus de produção e mais diversificados quanto aos contextos de localização (Marques et al., 2015, 2016). Como consequência tem vindo a ocorrer uma transição acelerada na produção de conhecimento do Modo 1, de tipo disciplinar e assente na investigação universitária de base, para a aplicação de conhecimento e solução de problemas baseada em conhecimento, numa base transdisciplinar (Modo 2) (Gibbons et al., 1994; Nowotny et al., 2001, 2003, 2006). Mais recentemente emergiu a necessidade de existir uma arquitetura de governança que potencie os processos de aprendizagem top-down (envolvendo universidades, indústria e governo) e bottom-up, incorporando as necessidades da sociedade civil (Modo 3 de produção de conhecimento) (Carayannis \& Campbell, 2012). Esta mudança facilita a coevolução dos contextos de produção, aplicação e utilização desse conhecimento e também da inovação (económica).

As parcerias e colaborações entre universidades, instituições públicas e empresas, o designado Triple Helix (Etzkowitz \& Leydesdorff, 1995, 1997, 2000; Etzkowitz, 2008), são cada vez mais valorizadas no processo de produção e valorização do conhecimento. A estes atores pertencentes a diferentes esferas de ação, junta-se a esfera da sociedade civil (quarta hélice), sendo que nos últimos anos tem vindo ser incorporada a dimensão ambiental traduzindo a quinta hélice do modelo de inovação (Carayannis \& Campbell, 2012). Globalmente, e numa visão evolucionista, constituem o ecossistema de inovação (Carayannis \& Campbell 2012). Como consequência, nesta abordagem ecossistémica da inovação (económica) sai reforçado o papel das redes interativas na estruturação dos processos de inovação territorializados. Daí a importância de ser necessário refletir os processos de governança e de participação tendo em vista o reforço das redes de inovação económica (com mais eficiência e eficácia) (Marques et al. 2015, 2016).

As redes de conhecimento científico entre investigadores e instituições são encaradas como a melhor aposta para a produção e difusão do conhecimento, ao criar parcerias entre universidades, institutos de ensino superior, laboratórios, centros de I\&D e empresas (Wagner, 2008). Muitas vezes, estas parcerias são responsáveis pela criação de novas empresas, que acabam por comercializar novos produtos, reconhecendo-se a importância da valorização económica do conhecimento (Etzkowitz, 2000; Goldstein, 2008).

Utilizando dados dos projetos de investigação do Horizonte 2020, pretende-se avaliar a configuração das redes estabelecidas, tendo em atenção as instituições/organizações envolvidas e a intensidade e expressão territorial das relações identificadas. Privilegia-se a análise de redes sociais e a leitura dos resultados numa dupla dimensão, topológica e territorial. Em paralelo, e para perceber as lógicas da estratégia de investigação da Universidade de Coimbra caracteriza-se o potencial das unidades de I\&D (recursos humanos, participação em projetos, resultados da investigação). A leitura territorial visa identificar as novas dinâmicas associadas à missão da universidade na qualificação regional no quadro da especialização inteligente e da geografia das redes do conhecimento.

\section{UNIVERSIDADE DE COIMBRA: INVESTIGAÇÃO E PARTICIPAÇÃO EM REDES DE CONHECIMENTO}

O crescimento económico e futuro da Europa impulsionaram o investimento em investigação e inovação consubstanciado em programas de financiamento competitivo que assentam no estímulo da excelência científica europeia. A implementação de projetos financiados neste âmbito tem vindo a refletir o esforço concertado da ciência e da indústria na busca de soluções para diversos desafios que, ao integrarem a investigação e a inovação, procuram contribuir para a melhoria da vida das pessoas, para a proteção do ambiente, bem como para a sustentabilidade e competitividade da indústria europeia. À semelhança dos programas-quadro anteriores, o Horizonte 2020 afigura-se como uma estratégia da Europa para um crescimento inteligente, sustentável e inclusivo que assenta em três pilares, designadamente: a excelência científica, a liderança industrial e os desafios societais (UE, 2014).

As universidades e instituições de ensino superior assumem-se como fundamentais para a dinamização de cidades e regiões e para a definição de novas estratégias de desenvolvimento, quer considerando as dimensões do ensino e formação de recursos humanos, quer a partir das diferentes unidades de I\&D que constituem os departamentos e institutos (Fernandes 2008). Em Portugal destacam-se três importantes polos universitários (Lisboa, Porto e Coimbra) responsáveis pela maior parte da oferta de ensino superior, bem como pela atividade de 
I\&D e inovação. Dos 322 estabelecimentos de ensino superior identificados pelo Observatório para a Ciência e Ensino Superior, 85 estão localizados em Lisboa, 67 no Porto e 22 em Coimbra. Considerando os estabelecimentos de ensino superior universitário de natureza pública, a oferta integra cerca de 86 estabelecimentos a nível nacional. As Universidades de Lisboa (UL) e Nova de Lisboa (UNL), reúnem um total de 28 estabelecimentos, seguindo-se a Universidade do Porto (UP) com 14 estabelecimentos e a Universidade de Coimbra (UC) com 10 estabelecimentos. Acresce que para a I\&D, inovação e tecnologia devem ser ainda consideradas as diferentes instituições com atividade de I\&D que operam tanto, em contexto das universidades e outros institutos de ensino superior, como noutras organizações governamentais e instituições privadas sem fins lucrativos. Neste contexto foram identificadas 1142 unidades com atividade de I\&D, correspondendo 591 ao setor do ensino superior (51,7\%), 467 ao domínio Estado (40,9\%) e 83 unidades a instituições privadas sem fins lucrativos (7,3\%) (Gama et al., 2017).

Considerando apenas as instituições com atividade de I\&D que integram a esfera do ensino superior (591), 64,6\% (382 unidades) integram o ensino superior público, sendo que as restantes pertencem ao ensino superior privado e politécnico. A localização destas 382 unidades de I\&D reflete o padrão de localização das instituições de I\&D nos concelhos de Lisboa (40,8\%), Porto (12,3\%), Coimbra (11,3\%), Braga-Guimarães (8,4\%) e Aveiro (4,2\%), reforçando a ideia de uma forte polarização das unidades de I\&D ancoradas às principais universidades públicas portuguesas.

A Universidade de Coimbra (UC) tem vindo sucessivamente a reforçar a sua presença no campo da inovação e da transferência de saberes, participando em inúmeras atividades e projetos de I\&D, desenvolvidas por docentes e investigadores (1778) oriundos das suas 8 Faculdades; Centros de Investigação (39 Unidades de I\&D financiadas e avaliadas pela FCT que abrangem as áreas das Ciências e da Tecnologia, das Ciências Sociais e das Humanidades) e Laboratórios Associados. Dados relativos ao ano letivo 2016/2017, indicam que, ao nível da investigação, a UC engloba 40 Unidades de investigação (63\% com avaliação externa excecional, excelente ou muito bom, estando envolvida em diversos projetos de investigação (311) ao longo desse período (http://www.uc.pt/ dados).

\section{REDES DE CONHECIMENTO DA UNIVERSIDADE DE COIMBRA: ATORES, REDES DE COLABORAÇÃO E TERRITÓRIO}

Reconhecendo que os projetos financiados pelo $\mathrm{H} 2020$ fomentam as parcerias entre universidades, associações, centros de investigação, instituições governamentais e empresas nacionais e internacionais, recorreu-se à metodologia de análise de redes sociais baseada na teoria dos grafos. Esta metodologia permite compreender as ligações entre os atores ou grupos intervenientes e as implicações dessas ligações para a estrutura e dinâmica da rede (Barnes, 1972; Barabási, 2002; Butts, 2008; Wal \& Boschma, 2009; Phelps et al., 2012; Scott, 2013).

A aplicação desta metodologia ao presente estudo, permitiu representar e analisar a rede de colaboração científica da Universidade de Coimbra com as outras instituições nos 36 projetos europeus $\mathrm{H} 2020$ identificados no momento do levantamento (março de 2018) (Tabela 1). O total de projetos representou um investimento de 131 milhões de euros. Na qualidade de promotora a UC mobilizou 35 participantes e cerca de 13 milhões de euros. O número médio de participantes por projeto foi de 4 e 13, no caso da UC ser promotora ou participante, respetivamente.

Tabela 1 - Projetos, custos, financiamento e participantes nos projetos H2O2O da Universidade de Coimbra.

\begin{tabular}{|ccccc|}
\hline Papel da UC & $\begin{array}{c}\text { Número de } \\
\text { projetos }\end{array}$ & $\begin{array}{c}\text { Valor total } \\
\text { dos projetos }\end{array}$ & Financiamento & Participantes \\
\hline $\begin{array}{c}\text { Projetos da UC como } \\
\text { promotora }\end{array}$ & 8 & 12944230,0 & 11875096,1 & 35 \\
\hline $\begin{array}{c}\text { Projetos da UC como } \\
\text { participante }\end{array}$ & 28 & 118186789,4 & 112237218,2 & 357 \\
\hline Total & 36 & $\mathbf{1 3 1} \mathbf{1 3 1 0 1 9 , 3}$ & $\mathbf{1 2 4 1 1 2 ~ 3 1 4 , 3}$ & $\mathbf{3 9 2}$ \\
\hline
\end{tabular}


Atendendo à dimensão das equipas envolvidas, mais de $50 \%$ dos projetos analisados apresentam menos de 10 participantes (20 projetos), sendo que 11 projetos (30,6\%) apresentam 15 e mais participantes (Tabela 2). A participação da Universidade de Coimbra faz-se de duas formas, como promotora ou como participante. Nos projetos em que a UC assume a liderança torna-se notório um menor número de organizações participantes. Em geral, dos oito projetos da UC como promotora, seis são constituídos por menos de cinco participantes, o que significa que a instituição tem desenvolvido a sua investigação recorrendo a um menor número de organizações. Acresce que existe um domínio de especialização (saúde) (Figura 1).

A rede de colaboração institucional da Universidade de Coimbra assume uma grande diversidade de atores (englobando cerca de 281 instituições relacionadas), sendo evidente que o grupo mais representativo são as universidades (39,1\%), seguindo-se os centros de investigação (26\%) e as empresas (26\%). Com uma menor representatividade na rede surgem as instituições governamentais $(5,3 \%)$ e as associações/fundações (3,6\%). Nos projetos em que a Universidade de Coimbra se assume como promotora é evidente um grande envolvimento com outras universidades (57,1\%), sublinhando-se a importância da proximidade institucional na justificação das rela-

Tabela 2 - Projetos segundo o número de participantes.

\begin{tabular}{|c|c|c|c|c|c|c|}
\hline \multirow{2}{*}{$\begin{array}{c}\text { Número de } \\
\text { participantes }\end{array}$} & \multicolumn{2}{|c|}{ Projetos } & \multicolumn{2}{|c|}{$\begin{array}{l}\text { Projetos da UC } \\
\text { como promotora }\end{array}$} & \multicolumn{2}{|c|}{$\begin{array}{l}\text { Projetos da UC } \\
\text { como participant }\end{array}$} \\
\hline & $\mathrm{n}^{\circ}$ & $\%$ & $\mathrm{n}^{\circ}$ & $\%$ & $\mathrm{n}^{\circ}$ & $\%$ \\
\hline 1 participante & 3 & 8,3 & 3 & 37,5 & 0 & 0,0 \\
\hline 3-5 participantes & 7 & 19,4 & 3 & 37,5 & 4 & 14,3 \\
\hline 6-10 participantes & 10 & 27,8 & 1 & 12,5 & 9 & 32,1 \\
\hline 11-14 participantes & 5 & 13,9 & 0 & 0,0 & 8 & 28,6 \\
\hline 15-20 participantes & 6 & 16,7 & 1 & 12,5 & 5 & 17,9 \\
\hline Mais de 20 participantes & 5 & 13,9 & 0 & 0,0 & 5 & 17,9 \\
\hline Total & 36 & 100 & 8 & 100 & 28 & 100 \\
\hline
\end{tabular}

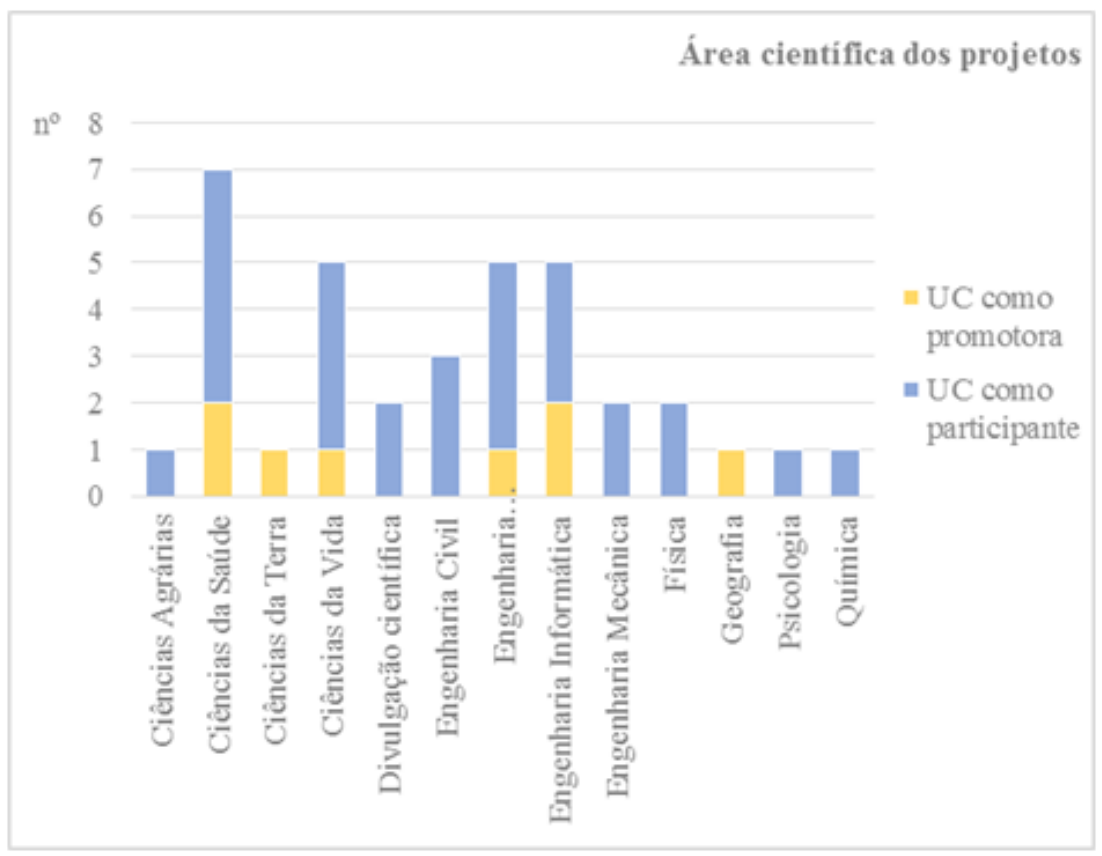

Figura 1 - Domínio científico dos projetos H2O2O com a participação da Universidade de Coimbra. 
ções estabelecidas (Tabela 3). Este aspeto comporta também uma menor diversidade de atores, visível também pelo menor número de organizações envolvidas na rede (28).

Numa leitura à origem dos atores envolvidos nestes projetos de investigação, destacam-se os atores estrangeiros, uma vez que dos 36 projetos, 23 são coordenados por equipas estrangeiras. Refere-se então que dos 281 atores presentes na rede, cerca de $90,7 \%$ são estrangeiros contra 9,3\% de origem portuguesa (Tabela 4). Dos atores estrangeiros, sublinha-se a grande representatividade de empresas (71, correspondendo a 97,3\%) e de universidades (100, correspondendo a 90,9\%).

Tabela 3 - Atores envolvidos no ecossistema de conhecimento da UC.

\begin{tabular}{|c|c|c|c|c|c|c|}
\hline \multirow[t]{2}{*}{ Tipologia } & \multicolumn{2}{|c|}{$\begin{array}{c}\text { Total de } \\
\text { atores }\end{array}$} & \multicolumn{2}{|c|}{$\begin{array}{c}\text { Atores nos } \\
\text { projetos da UC } \\
\text { como promotora }\end{array}$} & \multicolumn{2}{|c|}{$\begin{array}{c}\text { Atores nos } \\
\text { projetos da } \mathrm{UC} \\
\text { como participante }\end{array}$} \\
\hline & $\mathrm{n}^{\circ}$ & $\%$ & $\mathrm{n}^{\circ}$ & $\%$ & $\mathrm{n}^{\circ}$ & $\%$ \\
\hline Associações/Fundações & 10 & 3,6 & 1 & 3,6 & 9 & 3,6 \\
\hline Centros de Investigação & 73 & 26,0 & 3 & 10,7 & 70 & 27,7 \\
\hline Empresas & 73 & 26,0 & 5 & 17,9 & 68 & 26,9 \\
\hline Instituições governamentais & 15 & 5,3 & 3 & 10,7 & 12 & 4,7 \\
\hline Universidades & 110 & 39,1 & 16 & 57,1 & 94 & 37,2 \\
\hline Total & 281 & 100 & 28 & 100 & 253 & 100 \\
\hline
\end{tabular}

Tabela 4 - Atores envolvidos no ecossistema de conhecimento da UC segundo a origem.

\begin{tabular}{|c|c|c|c|c|c|c|}
\hline \multirow[t]{2}{*}{ Tipologia } & \multicolumn{2}{|c|}{$\begin{array}{c}\text { Total de } \\
\text { atores }\end{array}$} & \multicolumn{2}{|c|}{$\begin{array}{c}\text { Atores } \\
\text { estrangeiros }\end{array}$} & \multicolumn{2}{|c|}{$\begin{array}{c}\text { Atores } \\
\text { nacionais }\end{array}$} \\
\hline & $\mathrm{n}^{\circ}$ & $\%$ & $\mathrm{n}^{\circ}$ & $\%$ & $\mathrm{n}^{\circ}$ & $\%$ \\
\hline Associações/Fundações & 10 & 3,6 & 9 & 90,0 & 1 & 10,0 \\
\hline Centros de Investigação & 73 & 26,0 & 64 & 87,7 & 9 & 12,3 \\
\hline Empresas & 73 & 26,0 & 71 & 97,3 & 2 & 2,7 \\
\hline Instituições governament ais & 15 & 5,3 & 11 & 73,3 & 4 & 26,7 \\
\hline Universidades & 110 & 39,1 & 100 & 90,9 & 10 & 9,1 \\
\hline Total & 281 & 100 & 255 & 90,7 & 26 & 9,3 \\
\hline
\end{tabular}

Na metodologia de análise de redes sociais são valorizadas as medidas que procuram caraterizar a estrutura da rede e as relações entre os elementos (Tabela 5). De acordo com a classificação de Baur et al (2009), as medidas podem ser agrupadas ao nível da rede, dos elementos e dos grupos.

Tal como vimos a rede total exprime as relações entre os 281 atores, que entre si podem desenvolver cerca de 2600 ligações (Figura 2). Já a rede em que a Universidade de Coimbra assume a liderança, conta com 28 atores que estabelecem entre si 145 relações (Figura 3).

Ao nível da análise da rede são aplicadas medidas para analisar a estrutura global da rede, como sendo a distância geodésica, o número médio de graus de separação e a densidade. A distância geodésica máxima corresponde à distância mais longa de um nó a outro, sendo que a rede total apresenta o valor de 6 e a rede da UC como promotora evidencia uma maior proximidade entre os atores (2). O número médio de graus de separação, ou seja, o número médio de nós que separa cada instituição de uma outra, é de 2,15 e 1,57, respetivamente.

A densidade varia entre 0 e 1 e indica o grau de conexão dos vértices ou nós na rede. Quanto mais nós estiverem conectados de forma direta a outros nós, maior é a densidade. Neste caso, a rede total apresenta o valor de 0,06 como resultado da presença de um elevado número de instituições. 
Tabela 5 - Medidas de análise da rede de colaboração em projetos H2020 com a participação da UC.

\begin{tabular}{|c|c|c|c|}
\hline Medi das & Descri ção & Rede Total & $\begin{array}{l}\text { Rede UC como } \\
\text { promotora }\end{array}$ \\
\hline $\mathrm{N}^{\circ}$ de nós & Número de atores intervenientes na rede. & 281 & 28 \\
\hline $\begin{array}{l}\mathrm{N}^{\circ} \text { de } \\
\text { linhas/relaçóes }\end{array}$ & Número de relações existentes entre os diferentes atores. & 2600 & 145 \\
\hline $\begin{array}{l}\text { Distância geodésica } \\
\text { máxima }\end{array}$ & Distância mais longa de um nó a outro. & 6 & 2 \\
\hline $\begin{array}{l}\text { Número médio de } \\
\text { graus de separação }\end{array}$ & Número médio de nós que separa cada atorinstituição de & 2,15 & 1,57 \\
\hline Densidade & $\begin{array}{l}\text { Expressa a razão entre as relações existentes e as } \\
\text { possiveis. Quantos mais nós estiverem conectados de } \\
\text { forma direta a outros nós, maior é a densidade. Indica o } \\
\text { "grau" de conexão dos vértices na rede. }\end{array}$ & 0,06 & 0,38 \\
\hline Grau médio & $\begin{array}{l}\text { Corresponde ao número médio de intervenientes aos } \\
\text { quais cada nó se encontra ligado. }\end{array}$ & 16,11 & 10,21 \\
\hline Proximidade média & $\begin{array}{l}\text { Mede a proximidade de cada nó a todos os outros nós } \\
\text { com quem estabelece relações (grau de abrangência de } \\
\text { cada ator). Baseia-se na soma das distâncias dos } \\
\text { caminhos mais curtos. }\end{array}$ & 0,002 & 0,02 \\
\hline $\begin{array}{l}\text { Int ermediação } \\
\text { média }\end{array}$ & $\begin{array}{l}\text { Mede o grau de extensão na qual um nó se encontra } \\
\text { situado entre os outros nós da rede. Permite perceber a } \\
\text { centralidade, posição intermediária e prestigio de um } \\
\text { interveniente. }\end{array}$ & 162,06 & 8,43 \\
\hline $\begin{array}{l}\text { Coeficiente médio } \\
\text { de clusterizaçấo }\end{array}$ & $\begin{array}{l}\text { Quantifica quão conectado está um determinado vértice } \\
\text { com os seus "vizinhos". }\end{array}$ & 0,95 & 0,98 \\
\hline
\end{tabular}

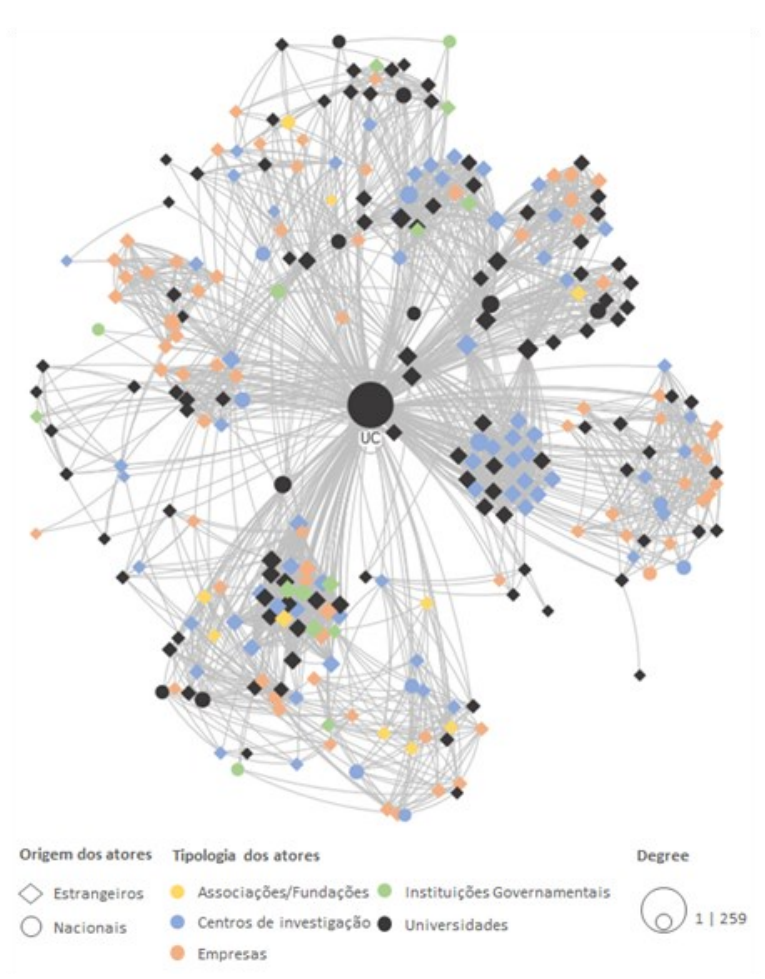

Figura 2 - Rede de colaboração da Universidade de Coimbra em projetos Horizonte 2020.

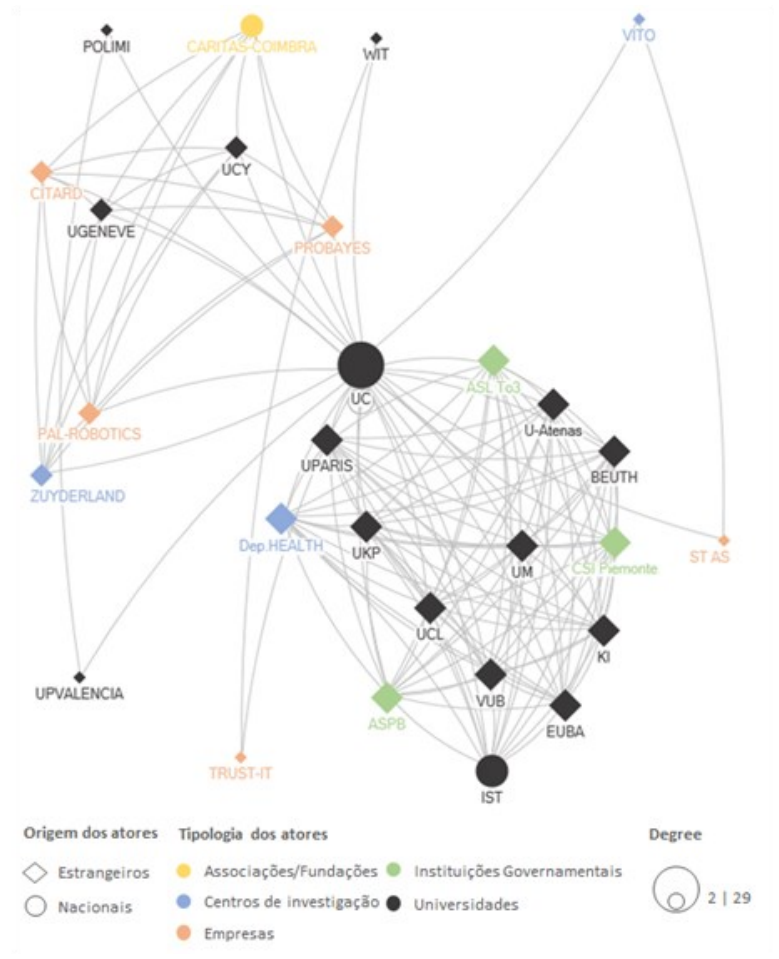

Figura 3 - Rede de colaboração da Universidade de Coimbra como promotora em projetos Horizonte 2020. 
Na análise ao nível dos elementos são valorizadas as medidas de centralidade (Freeman et al, 1979; Gómes et al.,2003), que determinam a importância relativa de um vértice no grafo: centralidade de grau (Degree Centrality), centralidade de proximidade (Closeness Centrality) e centralidade de intermediação (Betweenness Centrality).

O grau médio corresponde ao número médio de nós (organizações) aos quais cada nó da rede se encontra ligado. A rede total apresenta um valor mais elevado $(16,11)$, indicando uma rede alargada com muitas interações entre as instituições, enquanto que a rede da UC como promotora apresenta um valor menor $(10,21)$.

A medida de centralidade de grau, ao medir o número de conexões diretas de cada ator no grafo, dá excelentes indicações sobre a importância das relações de cada uma das instituições com as restantes.

Ao nível da centralidade de grau da rede total, destacam-se algumas organizações com valores muito acima da média, de que é o caso da Universidade de Coimbra, com 259 ligações diretas a outras unidades, uma vez que a recolha dos projetos se fez com base na participação desta instituição. Segue-se o Centre National de la Recherche Scientifique, com 44 ligações, o Stichting VU (42 ligações), a Universidade de Maastricht (38 ligações), a Universidade de Van Amsterdam (38 ligações), o Consiglio Nazionale Delle Ricerche (37 ligações) e a Universidade de Utrecht (36 ligações).

Já no caso da rede em que a Universidade de Coimbra é promotora, para além da UC (29 ligações), sobressaem a Agencia de Salut Publica de Barcelona (14 ligações), o Department of Health (14 ligações), a Universite Paris Ouest Nanterre La Defense (14 ligações), Instituto Superior Técnico (14 ligações) e a Universidade de Maastricht (14 ligações). Estas instituições, por apresentarem uma grande quantidade de contactos diretos, beneficiam de uma maior centralidade, sendo consideradas como as mais populares da rede.

A proximidade é uma medida que assenta na distância geodésica, ou seja, no comprimento do caminho mais curto que liga dois atores (Lemieux et al, 2004). Simboliza a proximidade de cada instituição a todas as outras com as quais estabelece relação. A proximidade média da rede total apresenta um valor muito baixo $(0,002)$, refletindo uma grande proximidade de cada instituição a todas as outras com as quais se encontra ligada.

A intermediação é outra medida de centralidade que permite medir o grau de extensão na qual um nó se encontra situado entre os outros nós da rede, sendo importante para aferir o prestígio das instituições e a sua capacidade para aceder e controlar o fluxo de informação pela posição intermediária que ocupam. Segundo Lemieux et al (2004), quanto mais um ator se encontrar numa posição intermediária, ou seja, quanto mais se encontrar numa situação em que os atores têm de passar por ele para chegar aos outros atores, maior capacidade de controlo terá sobre a circulação da informação entre esses atores. Nesta rede o valor médio é de 162,06, o que revela uma grande importância dos atores intermediários na rede. Já a rede da UC como promotora, em virtude do menor número de atores apresenta o valor de 8,43.

Por fim, ao nível da análise dos grupos, foi destacado o coeficiente de clusterização que quantifica quão conectado está um determinado vértice com os seus vizinhos (Hansen et al, 2011). Os valores calculados para as duas redes são semelhantes, 0,95 e 0,98, respetivamente.

Um último aspeto a valorizar diz respeito à identificação das relações espaciais da Universidade de Coimbra com os restantes territórios, analisada através da representação cartográfica de todas as unidades presentes na rede, bem como as relações potenciais entre a UC e as restantes organizações (Figuras 4 e 5).

No que diz respeito à rede de colaboração nos projetos em que a Universidade de Coimbra é promotora rapidamente se constata que a proximidade geográfica é pouco relevante para o estabelecimento de relações, sendo que a quase totalidade das relações são estabelecidas com organizações estrangeiras.

\section{NOTAS FINAIS}

A leitura das redes de colaboração científica constitui um excelente indicador para o delinear das políticas públicas, no sentido de reconhecer importância às redes existentes e fomentar o aparecimento de novas redes. Estas deverão ser vistas como mais-valias para a produção e difusão do conhecimento científico, capazes de promover processos de inovação determinantes para o desenvolvimento e aumento da capacidade competitiva dos territórios.

A análise sublinha a crescente participação da Universidade de Coimbra em redes de I\&D e inovação, quer como promotora quer como participante. Por outro lado, a análise mostra uma tendência de especialização na área científica das ciências da saúde. Um outro elemento indica a diversidade de atores envolvidos nos projetos de investigação considerando a importância das universidades e centros de investigação, mas também das empresas. As proximidades organizacional e institucional são importantes assim como a proximidade cognitiva, a 


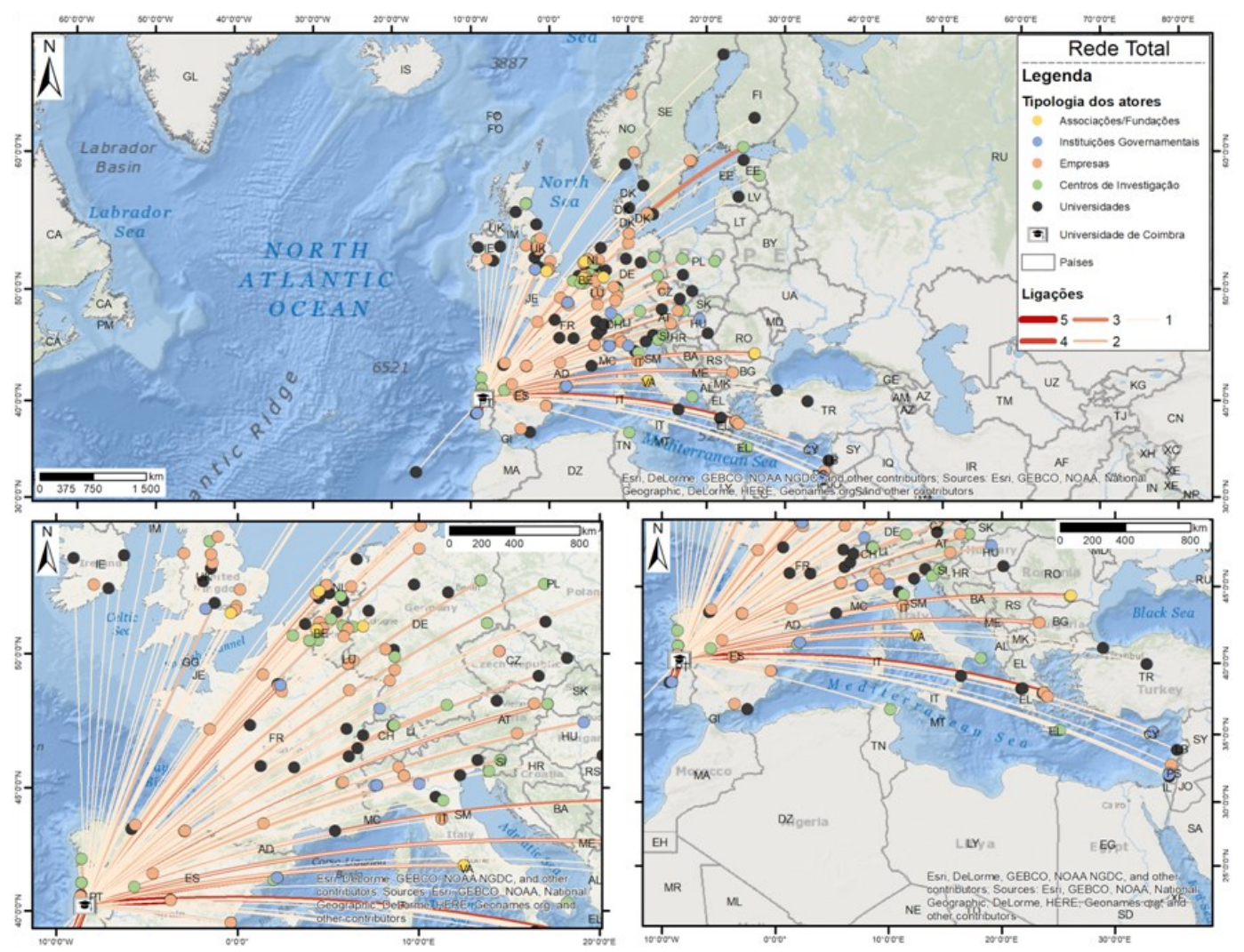

Figura 4 - Rede de colaboração da Universidade de Coimbra em projetos Horizonte 2020 (Rede Total



Figura 5 - Redes de colaboração da Universidade de Coimbra em projetos Horizonte 2020 (Rede UC promotora). 
procura de parceiros com as competências necessárias atendendo aos objetivos das colaborações. Neste sentido a proximidade geográfica assume um papel de menor importância no estabelecimento de parcerias.

Importa ainda destacar a crescente importância das parcerias com instituições e unidades de investigação internacionais e, em especial, com empresas estrangeiras.

A leitura da participação da UC em Projetos financiados pelo H2020 deixa antever um fortalecimento nas relações, assente na diversidade de atores (institucional e espacial), internacionalização e crescente participação de empresas que tem contribuído para o alargamento das redes ao longo dos anos.

\section{BIBLIOGRAFIA}

BARABÁSI, A. (2002). Linked-the new science of networks. Cambridge: Perseus Publishing.

BARNES, J. (1972). Social networks. Addison-Wesley Module in Anthropology, 26, 1-29.

BAUR, M., BRANDES, U., LERNER, J., \& WAGNER, D. (2009). Group-level analysis and visualization of social networks (pp. 330 -358). In J. Lerner, D. Wagner \& K. A. Zweig (Eds.), Algorithmics of Large and Complex Networks, Design, Analysis, and Simulation. Heidelberg: Springer

BRANDÃO, T. (2016). Shaping Portuguese science policy for the European horizon: The discourses of technological change. Technological Forecasting \& Social Change, 113, 168-184. doi:10.1016/j.techfore.2015.09.014

BUTTS, C. (2008). Social network analysis: A methodological introduction. Asian Journal of Social Psychology, 11, 13-41. doi:10.1111/j.1467-839X.2007.00241.x

CARAYANNIS, E. G., \& CAMPBELL, D. F. J. (2012). Mode 3 knowledge production in Quadruple Helix Innovation Systems. 21st -century democracy, innovation, and entrepreneurship for development. Springer Briefs in Business. New York: Springer. doi:10.1007/978-1-4614-2062-0

CE (2011). Livro Verde - Dos Desafios às Oportunidades: Para um Quadro Estratégico Comum de Financiamento da Investigação e Inovação da UE. Comissão Europeia. Consultado em http://www.qren.pt/np4/np4/?newsld=1334\&fileName=file879.pdf

ETZKOWITZ, H. (2008). The Triple Helix - University-Industry-Government-Innovation in Action. New York: Routledge. Consultado em https://ssrn.com/abstract=2480085

ETZKOWITZ, H., \& LEYDESDORFF, L. (1995). The Triple Helix University-Industry-Government Relations: A Laboratory for Knowledge-Based Economic Development. EASST Review 14,14-19.

ETZKOWITZ, H., \& LEYDESDORFF, L. (1997). Universities and the Global Knowledge Economy: A Triple Helix of UniversityIndustry-Government Relations. London: Pinter.

EtZkowitz, H., \& LeYdesdorfF, L. (2000). The Dynamics of Innovation: From National Systems and "Mode 2" to a Triple Helix of University-Industry-Government Relations. Research Policy, 29(2), 109-123. doi:10.1016/S0048-7333(99)00055-4

FERNANDES, R. (2008). Cidades e Regiões do Conhecimento: Do digital ao inteligente - Estratégias de desenvolvimento territorial. Coimbra: Universidade de Coimbra. Consultado em http://hdl.handle.net/10316/12399

FREEMAN, L., ROEDER, D., \& MULHOLLAND, R. (1979). Centrality in social networks: ii. experimental results. Social Networks, 2, 119-141. doi:10.1016/0378-8733(79)90002-9

GAMA, R., FERNANDES, R., \& BARROS, C. (2013) Redes de I\&D da Universidade de Coimbra: análise dos projetos de IC\&DT financiados pela Fundação para a Ciência e Tecnologia (FCT). Actas do IX Congresso da Geografia Portuguesa, 241-246.

GOLDSTEIN, H. (2010). The 'entrepreneurial turn' and regional economic development mission of universities. Annals of Regional Science, 44, 83-109. doi:10.1007/s00168-008-0241-z

GÓMES, D. et al. (2003). Centrality and power in social networks: a game theoric approach. Mathematical Social Sciences, 46, 27-54. doi:10.1016/S0165-4896(03)00028-3

HANSEN, D., SHNEIDERMAN, B., \& SMITH, M. (2011). Analyzing Social Media Networks with NodeXL. USA: Elsevier.

HEITOR, M. V., \& HORTE, H. (2011). Science and Technology in Portugal: From Late Awakening to the Challenge of Knowledge Integrate Communities. In G. Neave \& A. Amaral (Eds.), Higher Education in Portugal 1974-2009 A Nation, a Generation (pp. 179-226). Dordrech, Heildelberg, London, New York: Springer.

HENNEMANN, S., \& LIEFNER, I. (2015). Global Science Colaboration. In D. Archibugi \& A. Filippetti (Eds.), The Handbook of Global Science, Technology, and Innovation (pp. 343-36). Chichester: John Wiley \& Sons, Ltd, 3.

LEMIEUX, V., \& OUIMET, M. (2004). Análise Estrutural das Redes Sociais. Epistemologia e Sociedade. Lisboa: Instituto Piaget. doi:10.1080/13662719400000002 
LUNDVALL, B.-Å., \& JOHNSON, B. (1994). The learning economy. Journal of Industry Studies, 1(2), December, 23-42.

MAISONOBE, M., ECKERT, D., GROSSETTI, M., JÉGOU, L., \& MILARD, B. (2016). The world network of scientific collaborations between cities: domestic or international dynamics? Journal of Informetrics, 10, 1025-1036. doi:10.1016/ j.joi.2016.06.002

MARQUES, T., SANTOS, H., \& RIBEIRO, P. (2015). Redes de inovação económica ancoradas no Arco Metropolitano de Lisboa (2007-2013). In J. M. F. Ribeiro, F. Moura, \& J. Chorincas, Uma Metrópole para o Atlântico (557-590). Lisboa: Fundação Calouste Gulbenkian.

MARques, T., SANtos, H., \& Ribeiro, P. (2016). Redes de inovação económica ancoradas na Região Centro (2007-2015). In J. M. F. Ribeiro, F. Moura, \& J. Chorincas, Portugal no Centro (464-501). Lisboa: Fundação Calouste Gulbenkian.

NOWOTNY, H., SCOTT, P., \& GIBBONS, M. (2001). Re-thinking science. Knowledge and the public in an age of uncertainty. Cambridge: Polity Press.

NOWOTNY, H., SCOTT, P., \& GIBBONS, M. (2003). Mode 2 Revisited: The New Production of Knowledge. Minerva, 41, 179194. Consultado em http://www.jstor.org/stable/41821245

NOWOTNY, H., SCOTT, P., \& GIBBONS, M. (2006). Re-Thinking Science: Mode 2 in Societal Context. In E. G. Carayannis \& D. F. J. Campbell (Eds.), Knowledge Creation, Diffusion, and Use in Innovation Networks and Knowledge Clusters. A Comparative Systems Approach across the United States, Europe and Asia (pp. 39-51). Westport, Connecticut: Praeger.

PATRÍCIO, M. (2010). Science Policy and the Internationalisation of Research in Portugal. Journal of Studies in International Education, 14(2), 161-182. doi:10.1177/1028315309337932

PHELPS, C., HEIDL, R., \& WADHWA, A. (2012). Knowledge, Networks, and Knowledge Networks. A review and Research Agenda. Journal of Management, 38(4), 1115-1166. doi:10.1177/0149206311432640

SCOTT, J. (2013). Social Network analysis. London: Sage.

WAL, A., \& BOSCHMA, R. (2009). Applying social network analysis in economic geography: framing some key analytic issues. The Annals of Regional Science, 43(3), 739-756. doi:10.1007/s00168-008-0258-3 Wagner, C. (2008). The New Invisible College - Science for Development. Washington: The Brookings Institution Press.

WILSDON, J. et al. (2011). Knowledge, networks and nations: global scientific collaboration in the 21st century. Royal Society Policy document 03/11. London: The Royal Society.

UNIÃO EUROPEIA (2014). Horizon 2020 em breves palavras: o programa quadro de investigação e inovação da UE. Luxemburgo: Serviço das Publicações Oficiais das Comunidades Europeias. doi:10.2777/82557

Este artigo foi desenvolvido no âmbito do projeto POCI-01-0145-FEDER- 031686 cofinanciado pelo Programa - Operacional Competitividade e Internacionalização (POCI), através do Portugal 2020 e do Fundo Europeu de Desenvolvimento Regional (FEDER) e por fundos nacionais através da FCT - Fundação para a Ciência e Tecnologia. 